This is the accepted manuscript made available via CHORUS. The article has been published as:

\title{
Camelback-shaped band reconciles heavy-electron behavior with weak electronic Coulomb correlations in superconducting TINi_\{2\}Se_\{2\}
}

N. Xu, C. E. Matt, P. Richard, A. van Roekeghem, S. Biermann, X. Shi, S.-F. Wu, H. W. Liu, D. Chen, T. Qian, N. C. Plumb, M. Radović, Hangdong Wang, Qianhui Mao, Jianhua Du, Minghu Fang, J. Mesot, H. Ding, and M. Shi

Phys. Rev. B 92, 081116 - Published 24 August 2015

DOI: 10.1103/PhysRevB.92.081116 


\title{
Camelback-shaped band reconciles heavy electron behavior with weak electronic Coulomb correlations in superconducting $\mathrm{TINi}_{2} \mathrm{Se}_{2}$
}

\author{
N. Xu, ${ }^{1,2, *}$ C. E. Matt, ${ }^{1,3}$ P. Richard, ${ }^{4,5}$ A. van Roekeghem, ${ }^{4,6}$ S. Biermann,,${ }^{6,7,8}$ X. Shi, ${ }^{1,4}$ \\ S.-F. Wu ${ }^{4}$ H. W. Liu, ${ }^{4}$ D. Chen, ${ }^{4}$ T. Qian, ${ }^{4}$ N. C. Plumb,${ }^{1}$ M. Radović, ${ }^{1,9}$ Hangdong Wang, ${ }^{10}$ \\ Qianhui Mao, ${ }^{10}$ Jianhua Du, ${ }^{10}$ Minghu Fang, ${ }^{10,11}$ J. Mesot,,${ }^{1,2,3}$ H. Ding, ${ }^{4,5}$ and M. Shi ${ }^{1, \dagger}$ \\ ${ }^{1}$ Swiss Light Source, Paul Scherrer Insitut, CH-5232 Villigen PSI, Switzerland \\ ${ }^{2}$ Institute of Condensed Matter Physics, École Polytechnique Fédérale de Lausanne, CH-1015 Lausanne, Switzerland \\ ${ }^{3}$ Laboratory for Solid State Physics, ETH Zürich, CH-8093 Zürich, Switzerland \\ ${ }^{4}$ Beijing National Laboratory for Condensed Matter Physics, \\ and Institute of Physics, Chinese Academy of Sciences, Beijing 100190, China \\ ${ }^{5}$ Collaborative Innovation Center of Quantum Matter, Beijing, China \\ ${ }^{6}$ Centre de Physique Théorique, Ecole Polytechnique, CNRS-UMR7644, 91128 Palaiseau, France \\ ${ }^{7}$ Collège de France - 11 place Marcelin Berthelot, 75005 Paris, France \\ ${ }^{8}$ Kavli Institute for Theoretical Physics, University of California - Santa Barbara, CA 93106, USA \\ ${ }^{9}$ SwissFEL, Paul Scherrer Institut, CH-5232 Villigen PSI, Switzerland \\ ${ }^{10}$ Department of Physics, Zhejiang University, Hangzhou 310027, China \\ ${ }^{11}$ Collaborative Innovation Center of Advanced Microstructures, Nanjing University, Nanjing 210093, China
}

(Dated: May 14, 2015)

\begin{abstract}
Combining photoemission spectroscopy, Raman spectroscopy and first-principles calculations, we characterize superconducting $\mathrm{TlNi}_{2} \mathrm{Se}_{2}$ as a material with weak electronic Coulomb correlations leading to a bandwidth renormalization of 1.4. We identify a camelback-shaped band, whose energetic position strongly depends on the selenium height. While this feature is universal in transition metal pnictides, in $\mathrm{TlNi}_{2} \mathrm{Se}_{2}$ it lies in the immediate vicinity of the Fermi level, giving rise to a pronounced van Hove singularity. The resulting heavy band mass resolves the apparent puzzle of a large normal-state Sommerfeld coefficient (Phys. Rev. Lett. 111, 207001) in this weakly correlated compound.
\end{abstract}

PACS numbers: 74.70.Xa, 74.25.Jb, 79.60.-i, 71.20.-b

The discovery of high-temperature superconductivity in iron-chalcogenide $\mathrm{A}_{x} \mathrm{Fe}_{2-y} \mathrm{Se}_{2}(\mathrm{~A}=\mathrm{Tl}, \mathrm{K}, \mathrm{Cs}, \mathrm{Rb})$ $[1,2]$ has raised a lot of attention because their unique Fermi surface (FS) topology without hole pocket [3-8] challenges the electron-hole quasi-nesting scenario as the main Cooper pairing force in the Fe-based superconducting materials [9]. However, due to the phase separation associated with Fe vacancy ordering $[10,11]$ in $\mathrm{A}_{x} \mathrm{Fe}_{2-y} \mathrm{Se}_{2}$, it is still unclear whether superconductivity in this compound is related to that of the other iron-based superconductors. Very recently, it has been found that the iso-structural material $\mathrm{TlNi}_{2} \mathrm{Se}_{2}$ with two more electrons on the $3 \mathrm{~d}$ shell shows superconductivity with $T_{c}=$ $3.7 \mathrm{~K}$ [12]. In contrast to $\mathrm{A}_{x} \mathrm{Fe}_{2-y} \mathrm{Se}_{2}$, X-ray diffraction results indicate that this system is homogeneous without $\mathrm{Ni}$ vacancy or phase separation, and stoichiometric $\mathrm{TlNi}_{2} \mathrm{Se}_{2}$ was confirmed by energy dispersive X-ray spectroscopy [12]. A large normal-state Sommerfeld coefficient has been attributed to heavy fermion behavior [12], and its square root relationship with magnetic field in the mixed state suggests $d$-wave wave paring symmetry. On the other hand, thermal conductivity measurements suggest multiple nodeless superconducting gaps [13]. Therefore, it is crucial to determine the electronic structure of $\mathrm{TlNi}_{2} \mathrm{Se}_{2}$ to understand the nature of the reported heavy fermions in this material and to establish possible connections with other unconventional superconductors.
In this letter, we present high-resolution angleresolved photoemission spectroscopy (ARPES) results on $\mathrm{TlNi}_{2} \mathrm{Se}_{2}$. Photon energy dependent measurements reveal that the electronic structure exhibits threedimensionality, with four bands crossing the Fermi level $\left(E_{F}\right)$. Our density functional theory (DFT) calculations, renormalized by a factor of 1.4, match the experimentally determined band structure very well, indicating weaker correlation effects in $\mathrm{TlNi}_{2} \mathrm{Se}_{2}$ than in its cousin $\mathrm{A}_{x} \mathrm{Fe}_{2-y} \mathrm{Se}_{2}$ [3]. We reveal that the flat top of one of the bands $(\gamma)$ exhibits an asymmetric back-bending feature resulting in a camelback shape near $E_{F}$ at $k=(0,0, \pi)$, the $\mathrm{Z}$ point of the first Brillouin zone (BZ). This shape is captured by our DFT calculations and seems to be a general feature of transition metal pnictides [14]. This $\gamma$ band forms four small FS lobes around the Z point, and between the lobes four flat parts resulting in a van Hove singularity (VHS) near $E_{F}$ are identified. This finding provides a natural explanation to the heavy electrons feature inferred from electronic specific heat and the upper critical field measurements [12] in this weakly correlated system. Indeed, in $\mathrm{TlNi}_{2} \mathrm{Se}_{2} \gamma$ takes on a value of $40 \mathrm{~mJ} / \mathrm{mol} \mathrm{K}^{2}$, which is 6 times larger than that of $\mathrm{BaFe}_{2} \mathrm{As}_{2}\left(5.6 \mathrm{~mJ} / \mathrm{mol} \mathrm{K}{ }^{2}\right)$ with stronger correlations (renormalisation factor $\sim 3[15]$ ).

Large single crystals of $\mathrm{TlNi}_{2} \mathrm{Se}_{2}$ were grown by the self-flux method [12]. ARPES measurements were per- 
formed at SIS beamline of Swiss Light Source, and at the 1-cubed ARPES end-station of BESSY with circular polarized photons ranging in $20-70 \mathrm{eV}$. The angular and energy resolutions were set to $0.2^{\circ}$ and $5 \sim 10 \mathrm{meV}$, respectively. Clean surfaces for the ARPES measurements were obtained by cleaving crystals in situ in a working vacuum better than $5 \times 10^{-11}$ Torr. We label the momentum $(k)$ values with respect to the $1 \mathrm{Ni}$ /unit cell $\mathrm{BZ}$, with the high symmetry points defined in Fig. 1(a). The Fermi level of the samples was referenced to that of a gold film evaporated onto the sample holder. Raman data have been recorded at room temperature using a 514.5 $\mathrm{nm}$ laser source and the single mode of a Horiba Jobin Yvon-T64000 micro-Raman spectrometer equipped with a CCD camera.

The Raman spectra in Fig. 1(b) show one $A_{1 g}$ mode $\left(177.9 \mathrm{~cm}^{-1}\right)$ and one $B_{1 g}\left(132.8 \mathrm{~cm}^{-1}\right)$ phonon, similar to the observation on the isostructural 122-ferropnictides [16]. As illustrated in the inset, the intensity of these peaks are in perfect concordance with the four-fold symmetry of the crystal and the spectra are exempt of extra modes, which suggests that our $\mathrm{TlNi}_{2} \mathrm{Se}_{2}$ samples are not phase-separated and do not show $\mathrm{Ni}$ vacancy ordering, in agreement with a previous study on $\mathrm{KNi}_{2} \mathrm{Se}_{2}$ [17]

Fig. 1(c) shows the wide-energy photoemission spectra of $\mathrm{TlNi}_{2} \mathrm{Se}_{2}$. Clear double-peak features due to spin-orbit interaction are identified for states of Se $3 d$ at binding energies $\left(E_{B}\right)=54.0$ and $54.9 \mathrm{eV}, \mathrm{Tl} 5 d$ at $E_{B}=12.9$ and $15.1 \mathrm{eV}$, and $\mathrm{Ni} 3 p$ at $E_{B}=66.3$ and $67.9 \mathrm{eV}$ (as shown in the inset). In Fig. 1(d), we plot the DFT band structure calculated for the experimental lattice parameters [12] and the height of Se from the Ni layer $\left(\mathrm{z}_{S e}\right)=0.355$ $\AA$. Four bands cross $E_{F}$ and exhibit non-negligible threedimensionality. The density of states (DOS) near $E_{F}$ is contributed mainly by the Ni $3 d$ states with a partial Se $4 p$ spectral weight, as seen from Fig. 1(d) and the calculated DOS in Fig. 1(e). We notice that one specific band $(\gamma)$ shows a back-bending camelback-shaped feature at the $\mathrm{Z}$ point (as shown in the dashed-line circle in Fig. $1(\mathrm{~d})$ ), leading to a flat band top sitting very close to $E_{F}$ and contributing to a sharp peak in the DOS (inset of Fig. 1(e)). This gives us a first indication that the heavy electrons reported in $\mathrm{TlNi}_{2} \mathrm{Se}_{2}$ may originate from this flat band top. This band results from the hybridization of the Ni- $d_{x y}$ and Se- $p_{z}$ orbitals and is a universal feature in transition metal pnictides and chalcogenides. The particular shape of its dispersion, with a back-bending feature near the $\Gamma$ and $\mathrm{Z}$ points, is due to the combination of two opposite binding effects. Indeed, while the interaction of Ni- $d_{x y}$ with Se- $\mathrm{p}_{z}$ is antibonding and causes the band to disperse upwards toward $\mathrm{Z}$ because of an increasing hybridization, the nearest-neighbor $\mathrm{Ni}-d_{x y}-\mathrm{Ni}-d_{x y}$ interaction becomes bonding near this point, which explains the energy minimum. In most iron pnictides, the resulting saddle points are found in the unoccupied states [14], but the originality of $\mathrm{TlNi}_{2} \mathrm{Se}_{2}$ is that the Fermi level
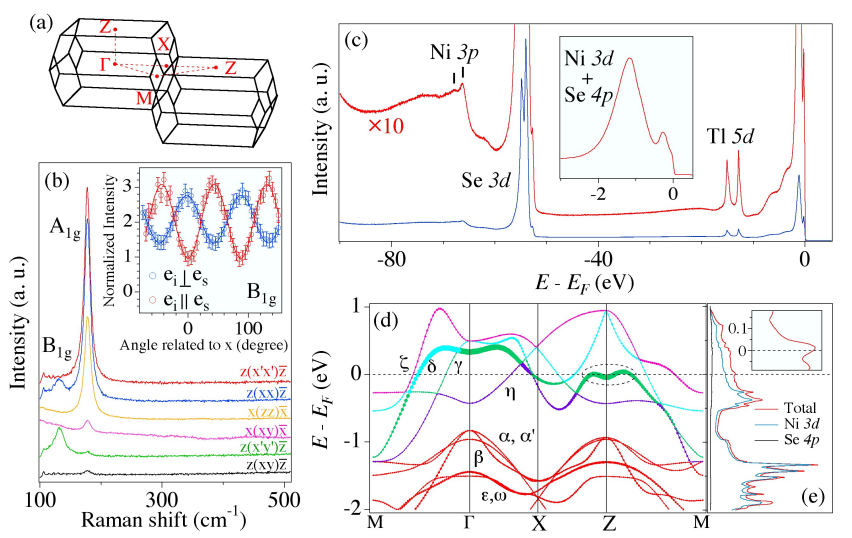

FIG. 1. (Color online): (a)Two adjacent BZs of $\mathrm{TlNi}_{2} \mathrm{Se}_{2}$. (b) Raman spectra recorded under several polarization configurations [18]. (c) Core level spectra of $\mathrm{TlNi}_{2} \mathrm{Se}_{2}$ recorded with $150 \mathrm{eV}$ photons. The inset is a close up of the valence band near $E_{F}$. (d) DFT band structure plotted along high symmetry lines. The bands near $E_{F}$ are colorized differently for a better visualization, and the line-width indicates the Se $4 p$ spectral weight. (e) DOS from DFT calculations.

crosses it.

In Figs. 2(a)-2(b), we plot normal emission ARPES intensities as a function of photon energy and their corresponding energy distribution curves (EDCs). Clear periodic variations of peak positions, especially for the $\gamma$ band near $E_{F}$ and the $\epsilon / \omega$ band at $E_{B} \sim 1.2 \mathrm{eV}$, are observed with tuning the $k_{z}$ value by changing the photon energy, indicating strong three-dimensionality of the band structure in $\mathrm{TlNi}_{2} \mathrm{Se}_{2}$. Using the nearly-free electron approximation with an inner potential of $17 \mathrm{eV}$, we estimate $h \nu=34 \mathrm{eV}$ for the $k_{z}=0$ plane, and $h \nu=$ $29 / 54 \mathrm{eV}$ for the $k_{z}=\pi$ planes. We plot the ARPES intensity along the $\Gamma-\mathrm{M}$ and Z-M directions in Figs. 2(c)$2(\mathrm{~d})$, which are recorded with $h \nu=34 \mathrm{eV}$ and $29 \mathrm{eV}$, respectively. The corresponding EDCs are shown in Figs. 2(e)-2(f). At first sight, the overall band structure of $\mathrm{TlNi}_{2} \mathrm{Se}_{2}$ shows great similarity with $\mathrm{BaFe}_{2} \mathrm{As}_{2}[19]$ and $\mathrm{BaCo}_{2} \mathrm{As}_{2}[20]$. The $\alpha, \alpha^{\prime}$ and $\beta$ hole-like pockets centred at the $\Gamma$ point in $\mathrm{BaFe}_{2} \mathrm{As}_{2}$ are fully filled in $\mathrm{TlNi}_{2} \mathrm{Se}_{2}$, with the bands topping $\sim 0.6 \mathrm{eV}$ below $E_{F}$. The $\gamma$ and $\delta$ electron-like pockets located at the $\mathrm{M}$ point in $\mathrm{BaFe}_{2} \mathrm{As}_{2}$ are more than half filled in $\mathrm{TlNi}_{2} \mathrm{Se}_{2}$ and form hole-like pockets at the $\Gamma$ point. One flat band $(\eta)$ sits $\sim 250 \mathrm{meV}$ below $E_{F}$ in $\mathrm{TlNi}_{2} \mathrm{Se}_{2}$, which is also observed near $E_{F}$ in $\mathrm{BaCo}_{2} \mathrm{As}_{2}$ [20, 21]. Besides all these states, the additional $\zeta$ band is observed in $\mathrm{TlNi}_{2} \mathrm{Se}_{2}$ around the $\mathrm{M}$ point. Our ARPES data suggest that $\mathrm{TlNi}_{2} \mathrm{Se}_{2}$ shares a universal band structure with $\mathrm{BaFe}_{2} \mathrm{As}_{2}$ and $\mathrm{BaCo}_{2} \mathrm{As}_{2}$, with a chemical potential shifted due to more $3 d$ electrons in $\mathrm{TlNi}_{2} \mathrm{Se}_{2}\left(3 d^{8.5}\right)$ than in $\mathrm{BaFe}_{2} \mathrm{As}_{2}\left(3 d^{6}\right)$ and $\mathrm{BaCo}_{2} \mathrm{As}_{2}$ $\left(3 d^{7}\right)$. This is indeed what is expected from our DFT calculations, which match the experimental data very well 

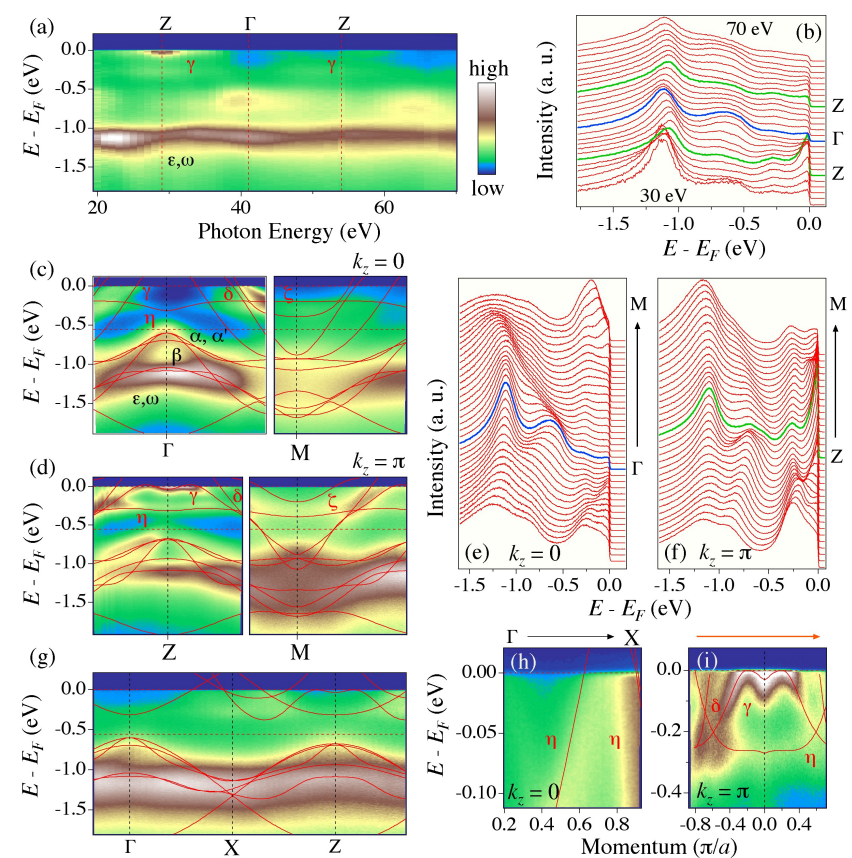

FIG. 2. (Color online) (a) Photon energy dependent ARPES intensity plot recorded at normal emission. (b) Corresponding EDC plot. (c)-(d) ARPES intensity plot along the $\Gamma-\mathrm{M}$ and Z-M directions. DFT results renormalized by a factor 1.4 are overlapped. (e)-(f) Corresponding EDC plots. (g) ARPES intensity plot along the $\Gamma-\mathrm{X}-\mathrm{Z}$ momentum path, with renormalized DFT. (h) Near- $\mathrm{E}_{F}$ zoom along the $\Gamma-\mathrm{X}$ direction. (i) Same as (h), with the location in k-space illustrated by the yellow arrow in Fig. 3(c).

with a renormalization factor of 1.4 as seen in Figs. 2(c)2(d). $\mathrm{TlNi}_{2} \mathrm{Se}_{2}$ can thus be viewed as heavily electron doped $\mathrm{TlFe}_{2} \mathrm{Se}_{2}\left(3 d^{6.5}\right)$, as we will discuss more later.

Similarly, we plot the ARPES intensity along $\Gamma-\mathrm{X}-\mathrm{Z}$ obtained with photon energy $h \nu=54 \mathrm{eV}$ in Fig. 2(g), which also shows a good agreement with the renormalized DFT calculations. In order to examine the details for the bands near $E_{F}$, high-resolution measurements have been recorded along $\Gamma-\mathrm{X}$ at the $k_{z}=0$ plane $(h \nu=41 \mathrm{eV})$. As shown in Fig. 2(h), the hole-like $\eta$ band crosses $E_{F}$, with the intensity enhanced due to the matrix element effect. Similarly, Fig. 2(i) shows the band structure in the $k_{z}=\pi$ plane $(h \nu=29 \mathrm{eV})$, cutting slightly off the $\mathrm{Z}-\mathrm{X}$ direction (as illustrated by the yellow arrow in Fig. $3(\mathrm{c})$ ), in order to well separate the $\gamma$ and $\delta$ bands. In agreement with DFT, the $\gamma, \delta$ and $\eta$ bands are observed, hybridizing with each other at their intersections.

In Figs. 3(a) and 3(b), we display the Fermi surface at $k_{z}=0$ and $\pi$, respectively. The corresponding DFT calculations (with the chemical potential slightly shifted upward by $7 \mathrm{meV}$ ) are also overlapped. The Fermi surface contours are relatively broad due to the threedimensionality of the system. Nevertheless, the overall
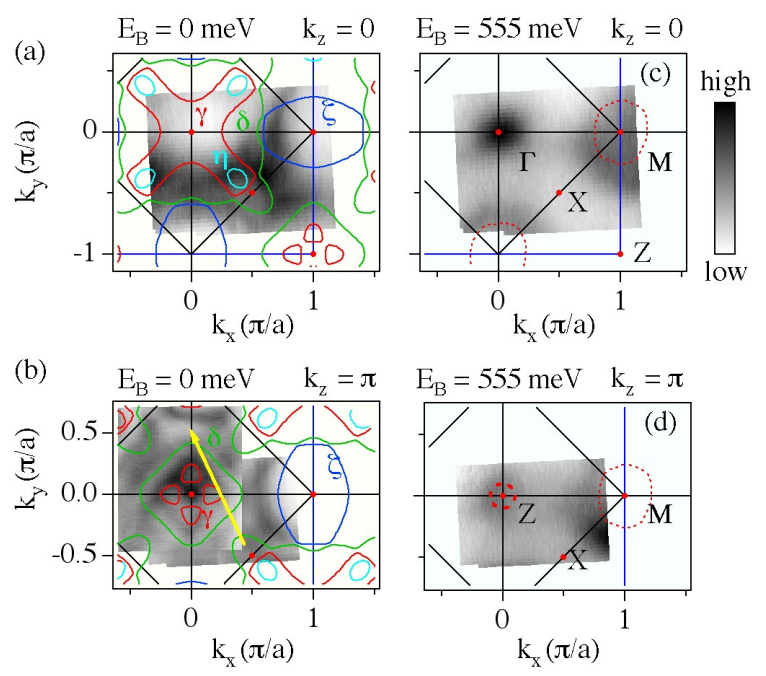

FIG. 3. (Color online) (a)-(b) FS mappings for the $k_{z}=0$ and $\pi$ planes, respectively, obtained by integrating the ARPES intensity within $E_{F} \pm 5 \mathrm{meV}$. (c)-(d) same as (a)-(b) but recorded $555 \mathrm{meV}$ below $E_{F}$.

features of the intensity map agrees with Fermi surface crossing expected by the calculations: the $\gamma$ and $\delta$ bands, which form a pair of degenerate electron-like pockets at the $\mathrm{M}$ point in $\mathrm{A}_{x} \mathrm{Fe}_{2-y} \mathrm{Se}_{2}[3-6]$, become hole-like pockets crossing $E_{F}$ at the $\Gamma$ point. The $\delta$ band crosses the $\mathrm{Z}$ point with a smaller $k_{F}$ and the $\gamma$ band creates four small lobes because of the back-bending feature discussed before. The $\eta$ band forms a small hole-like pocket near the $\mathrm{X}$ point, and the $\zeta$ band forms an electron-like pocket at the $\mathrm{M}$ point. In contrast, we find that the constant energy maps recorded at $550 \mathrm{meV}$ below $E_{F}$ for the $k_{z}=$ 0 and $\pi$ planes, which are, respectively, displayed in Figs. $3(\mathrm{c})$ and $3(\mathrm{~d})$, are quite similar to the FSs of $\mathrm{A}_{x} \mathrm{Fe}_{2-y} \mathrm{Se}_{2}$, on which superconducting gaps are observed below $T_{c}$ [6]. To illustrate this resemblance, we overlap on these figures the FSs obtained for $\mathrm{A}_{x} \mathrm{Fe}_{2-y} \mathrm{Se}_{2}$ in Ref. [6]. Similarly to the case of $\mathrm{BaFe}_{2} \mathrm{As}_{2}$ and $\mathrm{BaCo}_{2} \mathrm{As}_{2}, \mathrm{TlNi}_{2} \mathrm{Se}_{2}$ can be interpreted as a heavily electron doped $\mathrm{TlFe}_{2} \mathrm{Se}_{2}$ as a first approximation. Therefore, our results suggest that $\mathrm{TlFe}_{2} \mathrm{Se}_{2}$, the phase without vacancy ordering, is the superconducting compound of the iron-chalcogenide $\mathrm{Tl}_{x} \mathrm{Fe}_{2-y} \mathrm{Se}_{2}$, which is consistent with the STM results [10] and supports the conclusions derived from previous ARPES experiments on $\mathrm{AFe}_{2-x} \mathrm{Se}_{2}$ [3-8].

The significant reduction of electron correlation strength in $\mathrm{TlNi}_{2} \mathrm{Se}_{2}$ (renormalization factor of 1.4) as compared to $\mathrm{TlFe}_{2} \mathrm{Se}_{2}$ (renormalization factor of 2.5 [3]) is anticipated due to the increased filling of the electronic $3 d$ shell. Indeed, the stronger correlations in $\mathrm{BaFe}_{2} \mathrm{As}_{2}$ as compared to $\mathrm{BaCo}_{2} \mathrm{As}_{2}$ are driven by the lower band filling in the presence of strong Hund's coupling [20]. The mass enhancement due to correlation effects in $\mathrm{TlNi}_{2} \mathrm{Se}_{2}$ 

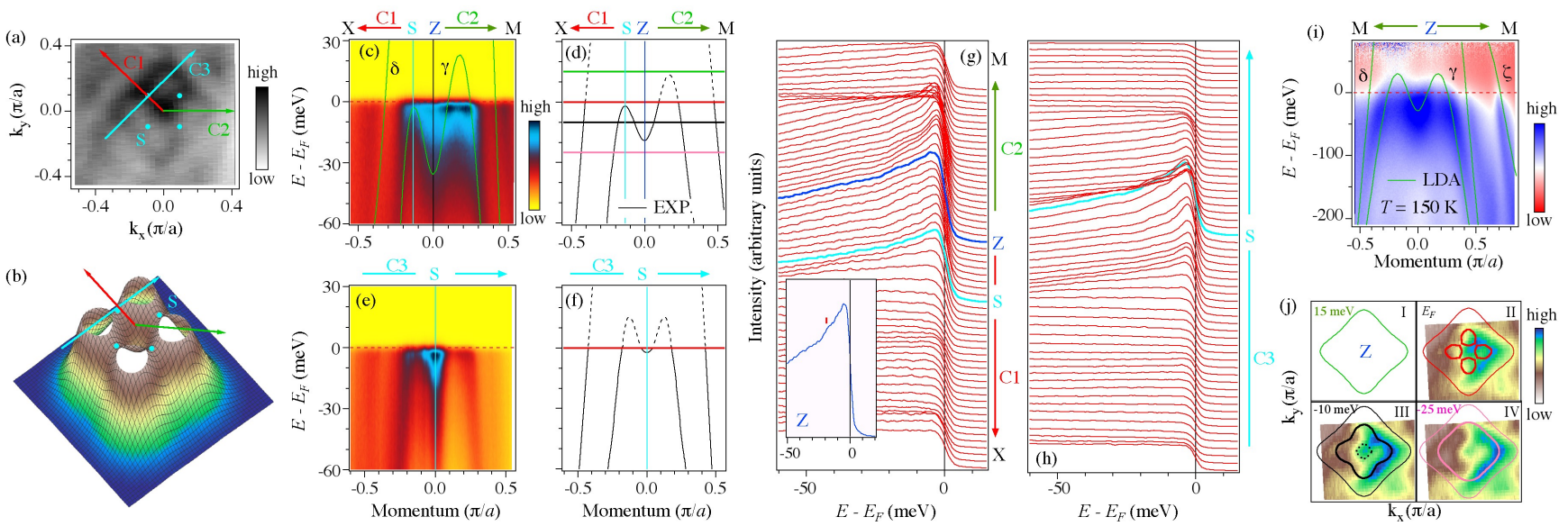

FIG. 4. (Color online) (a) FSs plot near the Z point. (b) Illustration of the band structure near the Z point. (c) ARPES results along C1-C2. (d) Corresponding extracted band dispersion. (e)-(f), Same as (c)-(d), but along C3. (g)-(h) EDC plots for C1-C2 and C3. The inset in (g) is the EDC taken at the Z point. (i) ARPES intensity plot taken at $T=150 \mathrm{~K}$. (j) Constant energy map contours taken near $E_{F}$.

is much smaller than that deduced from the Sommerfeld coefficient [12]. Here we suggest the large Sommerfeld coefficient to be related to the flat band near the chemical potential at the $\mathrm{Z}$ point. As seen from the result in Fig. 4(c) and the corresponding EDC plot in Fig. 4(g), which is recorded at $T=5 \mathrm{~K}$, the top of the $\gamma$ band sits at the S point, $\sim 2 \mathrm{meV}$ below the $E_{F}$ along the Z-X direction (C1 in Fig. 4(a)), and bends back with the band bottom at $\sim 18 \mathrm{meV}$ below the $E_{F}$ (indicated by the EDC taken at the $\mathrm{Z}$ point shown in the inset of Fig. 4(g)). Along the Z-M direction (C2 in Fig. 4(a)), the band top of $\gamma$ is slightly above $E_{F}$, leading the $\gamma$ band to double-cross $E_{F}$ and forming small lobes. The $\gamma$ band top along Z$\mathrm{M}$ is estimated at $\sim 15 \mathrm{meV}$ above $E_{F}$ from the high temperature data $(150 \mathrm{~K})$ divided by the Fermi function shown in Fig. 4(i). In Fig. 4(e), we plot the ARPES intensity along the momentum path passing through the $\mathrm{S}$ point and perpendicular to the $\mathrm{Z}-\mathrm{X}$ direction (C3 in Fig. 4(a)). The corresponding EDC plot is also shown in Fig. 4(h). The $\gamma$ band crosses $E_{F}$ four times (passing through two small hole-like lobes), and has a negative curvature at the $\mathrm{S}$ point along $\mathrm{C} 1$, as seen from Fig. 4(e), and the extracted band structure in Fig. 4(f). At the same time, the $\gamma$ band has a positive curvature at the $\mathrm{S}$ point along the perpendicular direction $\mathrm{C} 1$ as shown in Figs. 4(c)-4(d). Therefore, our data confirms the picture discussed before and in particular the camelback shape and the VHS at the S points. This can be better visualised in the band structure plotted in Fig. 4(b). In fact, this VHS is guaranteed by the asymmetry of the backbending feature as shown in Fig. 4(c) and the extracted band structure in Fig. 4(d) along the X-Z-M direction.

The existence of VHS very close to $E_{F}$, provides a natural explanation for the large Sommerfeld coefficient in this weakly correlated system $\mathrm{TlNi}_{2} \mathrm{Se}_{2}$. In the mean time, the FS topology of the $\gamma$ band changes dramatically when slightly tuning the chemical potential due to the VHS, as indicated in Fig. 4(j) I-IV. When we put the chemical potential $>15 \mathrm{meV}$ above $E_{F}$, only the $\delta$ band crosses at the $\mathrm{Z}$ point and forms a hole-like pocket. By putting the chemical potential at $2 \mathrm{meV}>\mathrm{E}_{B}>-15 \mathrm{meV}$, the $\gamma$ band crosses the chemical potential but only along the Z-M directions, forming four small hole-like lobes. When the chemical potential is setting in the range of $17 \mathrm{meV}>E_{B}>2 \mathrm{meV}$, the $\gamma$ band crosses the chemical potential twice both along the Z-M and Z-X directions, forming a small electron-like pocket inside a concentric hole-like pocket. Further shifting the chemical potential down to $E_{B}>17 \mathrm{meV}$, the inner electron pocket disappears, leaving two hole-like pockets at the $\mathrm{Z}$ point. All these Lifshitz transitions associated with VHS happen with the chemical potential varying by less than $30 \mathrm{meV}$, providing a possible explanation for why $T_{c}$ of $\mathrm{KNi}_{2} \mathrm{Se}_{2}$ $(\sim 0.8 \mathrm{~K})[22]$ is $\sim 4$ times smaller than that of $\mathrm{TlNi}_{2} \mathrm{Se}_{2}$. Because $\mathrm{K}$ is much more sensitive to air than Tl, a slight shift of chemical potential in $\mathrm{KNi}_{2} \mathrm{Se}_{2}$ is expected. The difference of DOS near $E_{F}$ due to this small chemical potential variation cannot explain $\sim 4$ times smaller $T_{c}$ in $\mathrm{KNi}_{2} \mathrm{Se}_{2}$. Most interestingly, since the strength of the hybridization of Ni- $d_{x y}$ with the Se- $p_{z}$ orbital controls the position of the saddle point with respect to $E_{F}$, the FS topology is highly sensitive to the height of the selenium atom over the Ni-Se plane. This may be related to the sudden $T_{c}$ drop under pressure recently reported in $\mathrm{TlNi}_{2} \mathrm{Se}_{2}$ [23].

It is worth mentioning that the end-member of the hole-doped $\mathrm{BaFe}_{2} \mathrm{As}_{2}$, the iron pnictide $\mathrm{KFe}_{2} \mathrm{As}_{2}$, shows some similarities with $\mathrm{TlNi}_{2} \mathrm{Se}_{2}$. A large Sommerfeld 
coefficient ( $\sim 94 \mathrm{~mJ} / \mathrm{mol} \mathrm{K}{ }^{2}$ ) [24] and nodal superconductivity have been reported in $\mathrm{KFe}_{2} \mathrm{As}_{2}$ [25-27] and $\mathrm{Ba}_{0.1} \mathrm{~K}_{0.9} \mathrm{Fe}_{2} \mathrm{As}_{2}$ [29]. A similar pressure effect has also been observed in $\mathrm{KFe}_{2} \mathrm{As}_{2}$ [26] and $\mathrm{TlNi}_{2} \mathrm{SeS}$ [23] and recently a VHS has been observed in $\mathrm{KFe}_{2} \mathrm{As}_{2}$ [30]. The VHS observed in the present work not only reconciles heavy electron behavior with weak electronic Coulomb correlations in superconducting $\mathrm{TlNi}_{2} \mathrm{Se}_{2}$, but also serves as a new key to understand the novel properties in nickeland iron- pnictide superconductors.

\section{ACKNOWLEDGMENTS}

This work was supported by the Swiss National Science Foundation (Grant No. 200021-137783), the SinoSwiss Science and Technology Cooperation (Project No. IZLCZ2138954), and MOST (Grant No. 2010CB923000, 2011CBA001000, 2011CBA00102, 2012CB821403 and 2013CB921703) and NSFC (11004232, 11034011/A0402, 11234014 and 11274362) from China, the National Science Foundation under Grant No. NSF PHY11-25915, IDRIS/GENCI Orsay under project 091393, and the European Research Council under project 617196. The work in ZJU was supported by the Natural Science Foundation of China (Grants No. 11374261 and No. 11204059), and the National Basic Research Programs of China (No. 2011CBA00103, No. 2012CB821404, and No. 2015CB921004).

* nan.xu@psi.ch

$\dagger$ ming.shi@psi.ch

[1] Jiangang Guo et al., Phys. Rev. B 82, 180520(R) (2010).
[2] M.H. Fang et al., Europhys. Lett. 94, 27009 (2011).

[3] T. Qian et al., Phys. Rev. Lett. 106, 187001 (2011).

[4] Y. Zhang et al., Nature Mater. 10, 273 (2011).

[5] D. Mou et al., Phys. Rev. Lett. 106, 107001 (2011).

[6] X.-P. Wang et al., Europhys. Lett. 93, 57001 (2011).

[7] Z.-H. Liu et al., Phys. Rev. Lett. 109, 037003 (2012).

[8] X.-P. Wang et al., Europhys. Lett. 99, 67001 (2012).

[9] P. Richard et al., Rep. Prog. Phys. 74, 124512 (2011).

[10] W. Li et al., Nature Phys. 8, 126 (2012).

[11] Z. Wang et al., Phys. Rev. B 83, 140505(R) (2011).

[12] H. Wang et al., Phys. Rev. Lett 111, 207001 (2013).

[13] X. C. Hong et al., Phys. Rev. B 90, 060504(R) (2014).

[14] O.K. Andersen, L. Boeri, Annalen der Physik 523(1-2), 8 (2011).

[15] P. Richard et al., Phys. Rev. Lett. 104, 137001 (2010).

[16] A. M. Zhang and Q. M. Zhang, Chin. Phys. B 22, 087103 (2013).

[17] N. Lazarević et al., Phys. Rev. B 87, 144305 (2013).

[18] Here $\mathrm{x}^{\prime}$ and $\mathrm{y}^{\prime}$ are parallel to the $\mathrm{Ni}-\mathrm{Ni}$ bounding while $\mathrm{x}$ and $\mathrm{y}$ are at $45^{\circ}$ from these axes. The inset shows the evolution of the Raman intensity of the $B_{1 g}$ mode as a function of the angle between the incident polarization $e_{i}$ and the $\mathrm{x}$ axis, for $e_{i}$ parallel and perpendicular to the scattered light polarization $e_{s}$.

[19] H. Ding et al., J. Phys.: Condens. Matter 23, 135701 (2011).

[20] N. Xu et al., Phys. Rev. X 3, 011006 (2013).

[21] R.S. Dhaka et al., Phys. Rev. B 87, 214516 (2013).

[22] J. R. Neilson et al., Phys. Rev. B 86, 054512 (2012).

[23] S. K. Goh et al., Phys. Rev. B 90, 201105 (2014).

[24] M. Abdel-Hafiez et al., Phys. Rev. B 85, 134533 (2012).

[25] J.-Ph. Reid et al., Phys. Rev. Lett. 109, 087001 (2012).

[26] F. F. Tafti et al., Nature Phys. 9, 349 (2013).

[27] M. A. Tanatar et al., Phys. Rev. Lett. 104, 067002 (2010).

[28] T. Sato et al., Phys. Rev. Lett 103, 047002 (2009).

[29] N. Xu et al., Phys. Rev. B 88, 220508 (2013).

[30] Delong Fang et al., arXiv:1412.0945. 